American Journal of Applied Sciences 9 (7): 961-967, 2012

ISSN 1546-9239

(C) 2012 Science Publications

\title{
On Stability and Bifurcation of Solutions of Nonlinear System of Differential Equations for AIDS Disease
}

\author{
El-Marouf, S.A.A. \\ Department of Mathematics, Faculty of Science, \\ Taibah University, Madinahmonwarah, Kingdom of Saudi Arabia
}

\begin{abstract}
Problem statement: This study aims to discuss the stability and bifurcation of a system of ordinary differential equations expressing a general nonlinear model of HIV/AIDS which has great interests from scientists and researchers on mathematics, biology, medicine and education. The existance of equilibrium points and their local stability are studied for HIV/AIDS model with two forms of the incidence rates. Conclusion/Recommendations: A comparison with recent published results is given. Hopf bifurcation of solutions of an epidemic model with a general nonlinear incidence rate is established. It is also proved that the system undergoes a series of Bogdanov-Takens bifurcation, i.e., saddle-node bifurcation, Hopf bifurcation and homoclinic bifurcation for suitable values of the parameters.
\end{abstract}

Key words: Epedimic models, infectious disease, HIV/AIDS model, local stability, hopf bifurcation, bogdanov-takens bifurcation

\section{INTRODUCTION}

There has been considerable interest in disease of Human Immunodeficiency Virus (HIV) infection pandemic and so it receives (Bachar and Dorfmayr, 2004; McCluskey, 2003; Elaiw, 2010; Hethcote et al., 1989; Hsieh and Chen, 2004; LaSalle, 1976; Moghadas and Gumel, 2003; Naresh et al., 2011) and references therein. There was an important report given by UN AIDS conclude that a number of more than 34 million population living with HIV by the end of the year 2003 and alos the AIDS is growing rapidly. The HIV/AIDS disease is a danger problem in poor countries. The competition between the human immunodeficiency virus and the human immune system has widely been studied (Mann and Tarantola, 1998). Mathematical and Statistical models have been proven valuable in understanding the dynamics of HIV/AIDS infection amongst a population or between interacting countries with treatment and or change of behavior (Mann and Tarantola, 1998). Recently, numerous mathematical models have been developed to describe different phenomena about this disease. The mathematical modeling has proven to be effective in improving information about the character of HIV/AIDS. Perelson and Nelson (1999) proposed an ordinary differential equations model of cell-free viral spread of immunodeficiency virus HIV in a well-mixed compartment such as bloodstream. They divided the model into the components: Uninfected healthy CD+ Tcells, latently infected CD4+T-cells and free virus. This model is very important as inspiration for many models which obtained results established the importance of mathematical techniques in HIV/AIDS researches. Hethcote et al. (1992). Discussed time delay in the removed class to account the period of temporarily immunity. Culshaw and Ruan (2000) studied the interaction between infection of a CD4 +Tcell and the emission of viral particles on a cellular level. In order to obtain the effect of the time delay on the stability of the endemic steady state. In their very recent study, Cai et al. (2009) investigated an HIV/AIDS model with treatment. They established two infective stages. Using mathematical analysis. They discussed the global analysis of the spread of the HIV disease computed by the number $R_{0}$ (basic reproduction) by which they could classify stability of equilibrium points. In this study we assume that the total population is divided into a susceptible class of size $\mathrm{S}(\mathrm{t})$ and an infectious class before the onset of AIDS. Since it is well known (Mann and Tarantola, 1998) that the infection period is very long (more than or equal ten years), it is further divided into several cases. In this study, we are going to consider the case in which the HIV/AIDS model allows for some infected people to go from the symptomatic phase to the asymptomatic phase by all sorts of treatment ways. It is very important konwing that the infected 
individuals do not change are treated or not change their behavior no matter if they are treated or not.

Thus the average value of contact of an person is constant for all subset of population. The disease transfer from rate is constant from stage $\mathrm{I}_{1}-\mathrm{I}_{2}$ stage. It is also supposed that infected individual in the second stage $\mathrm{I}_{2}$ can go back by successful treatment to the first stage $I_{1}$ with rate $\gamma$. Here, we consider the treatment model with two infective stages in a general model than given by (Culshaw and Ruan, 2000; Perelson and Nelson, 1999). We consider the system Eq. 1:

$$
\begin{aligned}
& \frac{\mathrm{dS}}{\mathrm{dt}}=\alpha \mathrm{d}-\mathrm{a} \rho \mathrm{H}\left(\mathrm{I}_{1}, \mathrm{I}_{2}\right) \mathrm{S}-\mathrm{dS} \\
& \frac{\mathrm{dI} \mathrm{I}_{1}}{\mathrm{dt}}=\operatorname{a\rho H}\left(\mathrm{I}_{1}, \mathrm{I}_{2}\right) \mathrm{S}-\left(\mathrm{d}+\mathrm{k}_{1}\right) \mathrm{I}_{1}+\gamma \mathrm{I}_{2} \\
& \frac{\mathrm{dI}}{\mathrm{dt}}=\mathrm{k}_{1} \mathrm{I}_{1}-\left(\mathrm{d}+\mathrm{k}_{2}+\gamma\right) \mathrm{I}_{2}
\end{aligned}
$$

where, $\mathrm{N}=\mathrm{S}+\mathrm{I}_{1}+\mathrm{I}_{2}$ is the total active population size. This is a generalization of the work of Liming (Cai et al., 2009). Where they discussed the special case. Here $\mathrm{S}$ represents the number proportion of susceptible, $\mathrm{I}_{1}$ is the number proportion of infective of first stage of treatment and $I_{2}$ is the number proportion of infective of the second stage of treatment, $b$ is the birth rate constant, $\mathrm{d}$ is the natural death rate constant, $\alpha$ is the average number of contacts of an individual per unit of time, $\rho$ is the probability of disease transmission per contact by an infective in $\mathrm{I}_{1}$ and $\mathrm{k}_{1}$ and $\mathrm{k}_{2}$ are the transfer rate constants $\mathrm{I}_{1} \rightarrow \mathrm{I}_{2}$ and $\mathrm{I}_{1} \rightarrow$ a respectively, where $\mathrm{A}$ is the number of AIDS cases, $\alpha$ is the transfer rate constant $\mathrm{I}_{1} \rightarrow \mathrm{I}_{2}$ (successful treatment ) a $\mathrm{H}\left(\mathrm{I}_{1}, \mathrm{I}_{2}\right.$ ) is a nonlinear function on the average number of new infections per unite time (nonlinear incidence). This study is organized as follows. We start by discussing the existence of equilibrium points and their local stability using Routh-Hurwitz method. In next section we study the bifurcation of solutions of the system (1). The study end with a brief discussion.

Stability Properties: The total population size $N(t)$ is variable with $\mathrm{N}^{\prime}(\mathrm{t})=\mathrm{d}(\alpha-\mathrm{N})-\mathrm{k}_{2} \mathrm{I}_{2}$. In the absence of disease, the population size $\mathrm{N}$ approaches carrying capacity $\alpha$ the differential equation for $\mathrm{N}$ implies that solutions of (1) starting in the positive orthant $\mathrm{R}^{3}+$ defined by Eq. 2:

$$
\Gamma=\left\{\left(\mathrm{S}, \mathrm{I}_{1}, \mathrm{I}_{2}\right) \in \mathbb{R}_{+}^{3}: \mathrm{S}+\mathrm{I}_{1}+\mathrm{I}_{2} \leq \alpha\right\}
$$

The following is the linearization due to the system (1) Eq. 3 and 4:
$X^{\prime}=M X$, where $X=\left(\begin{array}{l}x_{1} \\ x_{2} \\ x_{3}\end{array}\right),\left(x_{1}, x_{2}, x_{3}\right) \in \mathbb{R}_{+}^{3}$

Where:

$$
M=\left(\begin{array}{ccc}
a \rho H-d & -a \rho \frac{\partial H}{\partial I_{1}} S & -a \rho \frac{\partial H}{\partial I_{2}} S \\
a \rho H & a \rho \frac{\partial H}{\partial I_{1}} S-\left(d+k_{1}\right) & -a \rho \frac{\partial H}{\partial I_{2}} S+\gamma \\
0 & k_{1} & -\left(d+k_{2}+\gamma\right)
\end{array}\right)
$$

It is clear from (1) that $\overline{\mathrm{P}}_{\circ}=(\alpha, 0,0)$ is a trivial equilibrium. The Varational matrix of (1) at $\overline{\mathrm{P}}_{\mathrm{o}}=(\alpha, 0$, 0 ) is given by Eq. 5:

$$
\underset{\overline{\mathrm{P}_{\circ}}}{\mathrm{M}}=\left(\begin{array}{ccc}
-\mathrm{d} & 0 & 0 \\
0 & -\mathrm{A} & \gamma \\
0 & \mathrm{k}_{1} & -\mathrm{B}
\end{array}\right)
$$

where, $\mathrm{A}=\left(\mathrm{d}+\mathrm{k}_{1}\right)>0$ and $\mathrm{B}=\left(\mathrm{d}+\mathrm{k}_{2}+\gamma\right)$. The eigenvalues are $\lambda_{1}=-d<0$ and $\lambda_{2}$ and $\lambda_{3}$ are given by:

$$
\lambda_{2,3}=\frac{-1}{2}(\mathrm{~A}+\mathrm{B}) \pm \frac{1}{2} \sqrt{(\mathrm{A}+\mathrm{B})^{2}-4\left(\mathrm{AB}-\mathrm{k}_{1} \gamma\right)} .
$$

Since $\lambda_{1}=-d<0, A>0$ and $B>0$, hence the disease-free point $\overline{\mathrm{P}}_{\circ}=(\alpha, 0,0)$ is locally asymptotically stable.

Now we study the non-zero equilibrium point $\mathrm{P}^{*}=\left(\mathrm{S}^{*}, \mathrm{I}_{1}{ }^{*}, \mathrm{I}_{2}{ }^{*}\right)$ of system $(1)$, where Eq. 6 :

$\mathrm{S}^{\star}=\frac{\alpha \mathrm{d}}{\mathrm{a} \rho \mathrm{H}\left(\mathrm{I}_{1}^{\star}, \mathrm{I}_{2}^{\star}\right)+\mathrm{d}}, \mathrm{I}_{1}^{\star}=\frac{\mathrm{a} \rho \mathrm{H}\left(\mathrm{I}_{1}^{\star}, \mathrm{I}_{2}^{\star}\right) \mathrm{S}^{\star}+\gamma}{\mathrm{d}+\mathrm{k}_{1}}$

$\mathrm{I}_{2}^{\star}=\frac{\mathrm{k}_{1} \mathrm{I}_{1}^{\star}}{\mathrm{d}+\mathrm{k}_{2}+\gamma}$

The Variational matrix of $(1.1)$ at $\mathrm{P}^{*}=\left(\mathrm{S}^{*}, \mathrm{I}_{1}{ }^{*}, \mathrm{I}_{2}{ }^{*}\right)$ is given by Eq. 7:

$\mathrm{M}_{\mathrm{P}^{\star}}=\left(\begin{array}{ccc}\mathrm{a} \rho \mathrm{H}^{\star}-\mathrm{d} & -\mathrm{a} \rho \mathrm{H}_{\mathrm{I}_{1}^{\star}} \mathrm{S}^{\star} & -\mathrm{a} \rho \mathrm{H}_{\mathrm{I}_{2}^{\star}} \mathrm{S}^{\star} \\ \mathrm{a} \rho \mathrm{H}^{\star} & \mathrm{a} \rho \mathrm{H}_{\mathrm{I}_{1}^{\star}} \mathrm{S}^{\star}-\left(\mathrm{d}+\mathrm{k}_{1}\right) & -\mathrm{a} \rho \mathrm{H}_{\mathrm{I}_{2}^{\mathrm{A}^{\star}}} \mathrm{S}^{\star}+\gamma \\ 0 & \mathrm{k}_{1} & -\left(\mathrm{d}+\mathrm{k}_{2}+\gamma\right)\end{array}\right)$

where, $\quad \mathrm{H}_{\mathrm{I}_{1}^{\star}}=\left.\frac{\partial \mathrm{H}}{\partial \mathrm{H}_{\mathrm{I}_{1}}}\right|_{\mathrm{I}_{1}=\mathrm{I}_{1}^{\star}}, \mathrm{H}_{\mathrm{I}_{2}^{\star}}=\left.\frac{\partial \mathrm{H}}{\partial \mathrm{H}_{\mathrm{I}_{2}}}\right|_{\mathrm{I}_{2}=\mathrm{I}_{2}^{\star}} \quad$ and $\mathrm{H}^{\star}=\mathrm{H}\left(\mathrm{I}_{1}^{\star}, \mathrm{I}_{2}^{\star}\right)$. We assume that $\mathrm{H}_{\mathrm{I}_{1}^{\star}}, \mathrm{H}_{\mathrm{I}_{2}^{\star}}$ and $\mathrm{H}^{*}$ are 
positive. Set $1_{1}=a \rho H^{*}, l_{2}=a \rho H_{1_{1}^{\star}} \mathrm{S}^{\star}$ and $l_{3}=a \rho H_{I_{2}^{\star}} \mathrm{S}^{\star}$. Thus the Jacobian matrix $\mathrm{M}_{\mathrm{P} *}$ becomes Eq. 8:

$\mathbf{M}_{\mathrm{P}^{\star}}=\left(\begin{array}{ccc}1_{1}-\mathrm{d} & -\mathrm{l}_{2} & -\mathrm{l}_{3} \\ \mathrm{l}_{1} & \mathrm{l}_{2}-\mathrm{A} & \mathrm{l}_{3}+\gamma \\ 0 & \mathrm{k}_{1} & -\mathrm{B}\end{array}\right)$.

The characteristic equation of $\mathrm{M}_{\mathrm{P}_{\mathrm{o}}}$ at $\mathrm{P} \circ$ is Eq. 9 and 10:

$\lambda^{3}+m_{1} \lambda^{2}+m_{2} \lambda+m_{3}=0$

Where:

$$
\begin{aligned}
& \mathrm{m}_{1}=\mathrm{d}-\mathrm{l}_{1}+\mathrm{A}-\mathrm{l}_{2}+\mathrm{B}, \\
& \mathrm{m}_{2}=\left(\mathrm{l}_{1}-\mathrm{d}\right)\left(\mathrm{l}_{2}-\mathrm{A}\right)+\mathrm{B}\left(\mathrm{d}-\mathrm{l}_{1}\right)+ \\
& \left(\mathrm{A}-\mathrm{l}_{2}\right) \mathrm{B}-\mathrm{k}_{1}\left(\mathrm{l}_{3}+\gamma\right)+\mathrm{l}_{1} \mathrm{l}_{2}, \\
& \mathrm{~m}_{3}=\left(\mathrm{l}_{1}-\mathrm{d}\right)\left(\mathrm{l}_{2}-\mathrm{A}\right) \mathrm{B}+\left(\mathrm{l}_{1}-\mathrm{d}\right) \\
& \left(\mathrm{l}_{3}+\gamma\right) \mathrm{k}_{1}+\mathrm{l}_{1} \mathrm{l}_{2} \mathrm{~B}+\mathrm{l}_{1} \mathrm{l}_{3} \mathrm{k}_{1}
\end{aligned}
$$

It follows from the Routh-Hurwitz criterion (ElMarouf and Alihaby, 2011; Rao, 1981) that $\mathrm{P}^{*}=$ $\left(\mathrm{S}^{*}, \mathrm{I}_{1}{ }^{*}, \mathrm{I}_{2}{ }^{*}\right)$ is locally asymptotically stable if $\mathrm{m}_{1}>0, \mathrm{~m}_{3}>$ 0 and $\mathrm{m}_{1} \mathrm{~m}_{2}-\mathrm{m}_{3}>0$, then we introduce the following result.

Theorem 2.1: Assume that the following conditions are satisfied:

$$
\begin{aligned}
& \left(\mathrm{A}_{1}\right) \mathrm{A}+\mathrm{B}+\mathrm{d}>\mathrm{l}_{1}+\mathrm{l}_{2} \\
& \left(\mathrm{~A}_{2}\right) \mathrm{l}_{1}\left(2 \mathrm{l}_{2} \mathrm{~B}+2 \mathrm{k}_{1}\left(\mathrm{l}_{3}+\gamma\right)\right)> \\
& \mathrm{AB}\left(\mathrm{l}_{1}-\mathrm{d}\right)+\mathrm{d}\left(\mathrm{l}_{2} \mathrm{~B}+\mathrm{k}_{1}\left(\mathrm{l}_{3}+\gamma\right)\right) \\
& \left(\mathrm{A}_{3}\right)(\mathrm{d}+\mathrm{A}+\mathrm{B})(\mathrm{x}-\mathrm{y})+\mathrm{dk}_{1}\left(\mathrm{l}_{3}+\gamma\right) \\
& >\left(\mathrm{l}_{1}+\mathrm{l}_{2}\right)(\mathrm{x}-\mathrm{y})+\mathrm{B}\left(2 \mathrm{l}_{1} \mathrm{l}_{2}+\mathrm{dA}\right)+\mathrm{l}_{1} \mathrm{k}_{1}\left(2 \mathrm{l}_{3}+\gamma\right)
\end{aligned}
$$

where, $\mathrm{x}=2 l_{1} \mathrm{l}_{2}+\mathrm{d}(\mathrm{A}+\mathrm{B})+\mathrm{AB}$ and $\mathrm{y}=\mathrm{l}_{1}(\mathrm{~A}+\mathrm{B})+\mathrm{l}_{2}$ $(d+B)+k_{1}\left(l_{3}+\gamma\right)$ Then the equilibrium point $P^{*}=\left(S^{*}\right.$ $\left.\mathrm{I}_{1}^{*}, \mathrm{I}_{2}^{*}\right)$ is locally asymptotically stable.

Now we choose $\gamma$ as the parameter of bifurcation for system (1.1). Let $\gamma_{c}$ be the value of $\gamma$ at which the characteristic Eq. 9 has two pure imaginary roots $\lambda_{1,2}$. From the above discussion we have the following theorem.

Theorem 2.2: Assume that the assumption (17) holds, thus at $\gamma=\gamma_{c}$, there exists one parameter family of periodic solutions bifurcating from the equilibrium point $\mathrm{P}^{*}=\left(\mathrm{S}^{*} \mathrm{I}_{1}{ }_{1}, \mathrm{I}_{2}{ }_{2}\right)$ with period $\mathrm{T}$, where $\mathrm{T} \rightarrow \mathrm{T} \circ$ as $\gamma$ $\rightarrow \gamma_{c}$ and where $\mathrm{T}_{0}=2 \pi / \omega_{0}=2 \pi / \sqrt{\mathrm{m}_{2}}$ and $\mathrm{m}_{2}$ is shown by (10).

Proof: It is clear that Eq. 9 has at least one real root $\lambda_{3}$, say, we have the following analysis Eq. 11:

$\left(\lambda-\lambda_{3}\right)\left[\lambda^{2}+\left(\lambda_{3}+m_{1}\right) \lambda+\left(\lambda_{3}^{2}+m_{1} \lambda_{3}+m_{2}\right)\right]=0$

Since, by (9) Eq. 12:

$\lambda_{1}+\lambda_{2}+\lambda_{3}=-\mathrm{m}_{1}$

also at $\gamma=\gamma_{c}$, we obtain Eq. 13:

$\lambda_{3}=-\mathrm{m}_{1}, \lambda_{1}=\bar{\lambda}_{2}$,
$\lambda_{1,2}=-\frac{1}{2}\left\{\left(\lambda_{3}+\mathrm{m}_{1}\right) \pm \sqrt{\left(\lambda_{3}+\mathrm{m}_{1}\right)^{2}-4\left(\lambda_{3}^{2}+\mathrm{m}_{1} \lambda_{3}+\mathrm{m}_{2}\right)}\right\}$ 14:

Thus, at $\gamma=\gamma_{c}>0$, we can rewrite Eq. 10 as Eq.

$\mathrm{D}_{\gamma}\left(\mathrm{m}_{1}\right)=\mathrm{m}_{1} \mathrm{~m}_{2}-\mathrm{m}_{3}$

Using $\mathrm{m}_{2}>0$ and $\mathrm{m}_{3}>0$, at $\gamma=\gamma_{\mathrm{c}}$, we get $\lambda_{3}=-\mathrm{m}_{1}<0$. At the critical value $\gamma=\gamma_{c}>0$, there is soultion of (14) which can be given by (11). Hence we obtain the equation of $\gamma$ as follows Eq. 15 and 16:

$-c_{1} \gamma^{2}-c_{2} \gamma+c_{3}=0$

Where:

$$
\begin{aligned}
& \mathrm{c}_{1}=-\left(\mathrm{d}-\mathrm{l}_{1}+\mathrm{d}^{2}+\mathrm{dk}_{1}-\mathrm{l}_{2}-\mathrm{k}_{1}\right) \\
& \mathrm{c}_{2}=-\left(2 \mathrm{~d}-\mathrm{l}_{1}+\mathrm{d}^{2}+\mathrm{dk}_{1}+\mathrm{k}_{2}-\mathrm{l}_{2}\right) \\
& \left(\mathrm{d}-\mathrm{l}_{1}+\mathrm{d}^{2}+\mathrm{dk}_{1}-\mathrm{l}_{2}-\mathrm{k}_{1}\right) \\
& -\left(\mathrm{l}_{1}-\mathrm{d}\right)\left(\mathrm{l}_{2}-\mathrm{d}^{2}-\mathrm{dk}_{1}\right)-\left(\mathrm{d}+\mathrm{k}_{2}\right) \\
& \left(\mathrm{d}-\mathrm{l}_{1}\right)-\left(\mathrm{d}^{2}+\mathrm{dk}_{1}-\mathrm{l}_{2}\right)\left(\mathrm{d}+\mathrm{k}_{2}\right) \\
& -\mathrm{k}_{1} 1_{3}+\mathrm{l}_{1} \mathrm{l}_{2}+\left(\mathrm{l}_{1}-\mathrm{d}\right)\left(\mathrm{l}_{2}-\mathrm{d}^{2}-\mathrm{dk}_{1}\right) \\
& \mathrm{c}_{3}=\left(2 \mathrm{~d}-\mathrm{l}_{1}+\mathrm{d}^{2}+\mathrm{dk}_{1}+\mathrm{k}_{2}-\mathrm{l}_{2}\right)\left[\left(\mathrm{l}_{1}-\mathrm{d}\right)\right. \\
& \left(\mathrm{l}_{2}-\mathrm{d}^{2}-\mathrm{dk}_{1}\right)\left(\mathrm{d}+\mathrm{k}_{2}\right)\left(\mathrm{d}-\mathrm{l}_{1}\right) \\
& \left.\left.\left(\mathrm{d}^{2}+\mathrm{d \textrm {k } _ { 1 }}-\mathrm{l}_{2}\right)\left(\mathrm{d}+\mathrm{k}_{2}\right)+\mathrm{k}_{1} \mathrm{l}_{3}+\mathrm{l}_{1} \mathrm{l}_{2}\right)\right] \\
& -\left(\mathrm{l}_{1}-\mathrm{d}\right)\left(\mathrm{l}_{2}-\mathrm{d}^{2}-\mathrm{dk}_{1}\right) \\
& \left.\left(\mathrm{d}+\mathrm{k}_{2}\right)+\left(\mathrm{l}_{1}-\mathrm{d}\right) \mathrm{l}_{3} \mathrm{k}_{1}+\mathrm{l}_{1} 1_{3} \mathrm{k}_{1}\right)
\end{aligned}
$$

Conversely, we assume that $\mathrm{m}_{1}>0, \mathrm{~m}_{3}>0$ and $\gamma>0$, then we can find the solution of Eq. 15 for $\gamma_{c}>0$. Also we know that $\mathrm{m}_{2}>0, \quad \lambda_{3}=-\mathrm{m}_{1}<0$ and $\lambda_{1,2}$ are conjugate imaginary. Now, we can choose both $1_{1}, l_{2}$ and $l_{3}$ to be 
sufficiently small and $\mathrm{d}, \mathrm{k}_{1}$ be sufficiently large. Then we have Eq. 17:

$\mathrm{d}\left(\mathrm{d}+\mathrm{k}_{1}+1\right)>\mathrm{l}_{1}+\mathrm{l}_{2}+\mathrm{k}_{1}$ Eq. 18:

But since by (15) and (16) $c_{1}>0 c_{2}>0$ and $c_{3}>0$

$\mathrm{D}_{\circ}\left(\mathrm{m}_{1}\right)=\mathrm{c}_{3}>0, \lim \mathrm{D}_{\gamma}\left(\mathrm{m}_{1}\right)=\infty$

Thus $\gamma_{c}$ is uniquely determined. Now, since by (11) $\lambda_{3}=-\mathrm{m}_{1}<0$ and Eq. 19:

$\mathrm{D}_{\gamma}\left(\mathrm{m}_{1}\right)=\mathrm{m}_{1} \mathrm{~m}_{2}-\mathrm{m}_{3}$

$=\left(\mathrm{m}_{1}+\lambda_{3}\right)\left(\lambda_{1} \lambda_{2}-\mathrm{m}_{1} \lambda_{1}\right)$

$\operatorname{sgnD}_{\gamma}\left(\mathrm{m}_{1}\right)=\operatorname{sgn}\left(\mathrm{m}_{1}+\lambda_{3}\right)$

Consequently we have Eq. 20 and 21:

$\operatorname{Re} \lambda_{1,2}=\frac{1}{2}\left(\mathrm{~m}_{1}+\lambda_{3}\right)<0$ for $\gamma>\gamma_{\mathrm{c}}$

$\operatorname{Re} \lambda_{1,2}>0$ for $\gamma<\gamma_{c}$

It follows from the above discussion that, when $\gamma$ is increased though $\gamma_{c}$, there exists a pair of complex conjugate imaginary eigenvalues $\lambda_{1,2}$ of the Variational

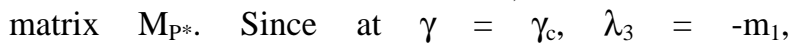
$\lambda_{1,2}= \pm \mathrm{i} \sqrt{\mathrm{m}_{2}}= \pm \mathrm{i} \omega_{\text {。 }}$ where it is clear that $\omega_{0}>0$. Now, since $\lambda_{1}=\bar{\lambda}_{2}$, for Eq. 22 :

$\operatorname{Re} \lambda_{2}=\frac{1}{2}\left(\lambda_{2}+\bar{\lambda}_{2}\right)=0$ at $\gamma=\gamma_{c}$

and by above discussion we see that $\operatorname{Re} \lambda_{2}>0$ for $\gamma<\gamma_{\mathrm{c}}$ and $\operatorname{Re} \lambda_{2}<0$ for $\gamma>\gamma_{c}$ thus Eq. 23:

$$
\begin{aligned}
& \left.\frac{\mathrm{d}}{\mathrm{d} \gamma}\left(\operatorname{Re} \lambda_{2}\right)\right|_{\gamma=\gamma_{\mathrm{c}}}=\left.\frac{-1}{2} \frac{\mathrm{d}}{\mathrm{d} \gamma}\left(\mathrm{m}_{1}+\lambda_{3}\right)\right|_{\gamma=\gamma_{\mathrm{c}}} \\
& =\left.\operatorname{Re}\left(\frac{\mathrm{d}}{\mathrm{d} \gamma} \lambda_{2}\right)\right|_{\gamma=\gamma_{\mathrm{c}}}<0 .
\end{aligned}
$$

This completes the proof.

Bogdanov-Takens Bifurcations: Now we onsider the system Eq. 24:

$$
\begin{aligned}
& \frac{d S}{d t}=\alpha d-a \rho H\left(S, I_{1}\right) S-d S, \\
& \frac{d I_{1}}{d t}=a \rho H\left(S, I_{1}\right) S-\left(d+k_{1}\right) I_{1}+\gamma I_{2},
\end{aligned}
$$

With the nonlinear incidence rate of the form $\mathrm{a} \rho \mathrm{H}\left(\mathrm{S}, \mathrm{I}_{1}\right)$. In order to translate the interior equilibria point $\mathrm{P}_{2}=\left(\mathrm{S}^{*}, \mathrm{I}_{1}{ }_{1}\right)$ to the origin, we set $\mathrm{X}=\mathrm{S}-\mathrm{S}^{*}$ and $\mathrm{Y}$ $=\mathrm{I}_{1}-\mathrm{I}_{1}{ }_{1}$, then the system (24) becomes Eq. 25:

$$
\begin{aligned}
& \frac{d X}{d t}=a_{11} X+a_{12} Y+f_{1}(X, Y) \\
& \frac{d Y}{d t}=a_{21} X+a_{22} Y+f_{2}(X, Y)
\end{aligned}
$$

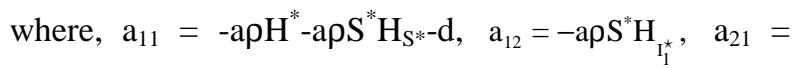
$a \rho H^{*}+a \rho S^{*} H_{S^{*}}, \quad a_{22}=a \rho S^{*} H_{I_{1}^{\star}}-\left(d+k_{1}\right)$, and $f_{1}(X, Y)$ and $\mathrm{f}_{2}(\mathrm{X}, \mathrm{Y})$ are smooth functions in $(\mathrm{X}, \mathrm{Y})$ of order at least two. Since we are interested in codimension 2bifurcation, we assume that:

$$
\left(\mathrm{A}_{1}\right) \quad 2 \mathrm{a} \rho \mathrm{S}^{*} \mathrm{H}_{\mathrm{I}_{1}^{\star}}<\left(\mathrm{d}+\mathrm{k}_{1}\right)
$$

Theorem 3.1: Assume that the assumption $\left(A_{1}\right)$ is satisfied. Then the equilibria point $\mathrm{P}_{2}=\left(\mathrm{S}^{*}, \mathrm{I}_{1}{ }_{1}\right)$ of (24) is a cusp of codimension 2, i.e., it is a BogdanovTakens singularity.

Proof: Using the assumption $\left(\mathrm{A}_{1}\right)$ the value of the determinant of the matrix Eq. 26:

$$
M_{\mathrm{P}_{2}=\left(\mathrm{S}^{*}, \mathrm{I}_{1}^{\star}\right)}=\left(\begin{array}{cc}
\mathrm{a} \rho \mathrm{H}^{*}-\mathrm{a} \rho \mathrm{S}^{*} \mathrm{H}_{\mathrm{s}^{*}} & -\mathrm{a} \rho \mathrm{S}^{*} \mathrm{H}_{\mathrm{I}_{1}^{\star}} \\
\mathrm{a} \rho \mathrm{H}^{*}+\mathrm{a}^{*} \mathrm{~S}_{\mathrm{s}^{*}} & \mathrm{a} \rho \mathrm{S}^{*} \mathrm{H}_{\mathrm{I}_{1}^{\star}}-\left(\mathrm{d}+\mathrm{k}_{1}\right)
\end{array}\right)
$$

is zero. Also, by the assumption $\left(\mathrm{A}_{1}\right)$ the matrix $\mathrm{M}$ has two zero eigenvalues. By the same transformation, we can write system (24) in the form Eq. 27:

$$
\begin{aligned}
& \frac{d X}{d t}=-\left(a \rho S^{*} H_{I_{1}^{*}}\right) Y-\frac{1}{2}\left[a \rho S^{*} H_{s^{*} s^{*}}+a \rho H_{s^{*}}\right] \\
& X^{2}+f_{3}(X, Y), \\
& \frac{d Y}{d t}=\frac{1}{2}\left[a \rho S^{*} H_{s^{*} s^{*}}+2 a \rho H_{s^{*}}\right] X^{2}+f_{4}(X, Y)
\end{aligned}
$$

where, $\mathrm{H}_{\mathrm{s}^{*} \mathrm{~s}^{*}}=\frac{\partial^{2} \mathrm{H}\left(\mathrm{S}^{*}, \mathrm{I}_{1}^{\star}\right)}{\partial \mathrm{S}^{2}}, \mathrm{f}_{3}(\mathrm{X}, \mathrm{Y})$ and $\mathrm{f}_{4}(\mathrm{X}, \mathrm{Y})$ are $\mathrm{C}^{\propto}$ functions at least of third order. After that, we discuss the normal form for the system (27) in the two dimensional and appling the center manifold theorem. Making the following affine transformation:

$$
\mathrm{x}=\mathrm{X} \text { and } \mathrm{y}=-\left(\operatorname{a\rho } \mathrm{S}^{*} \mathrm{H}_{\mathrm{I}_{1}^{\star}}\right) \mathrm{Y}
$$


system (27) can be written as Eq. 28:

$$
\begin{aligned}
& \frac{d x}{d t}=y-\frac{1}{2}\left[a \rho S^{*} H_{s^{*} s^{*}}+2 a \rho H_{s^{*}}\right] x^{2}+f_{5}(x, y), \\
& \frac{d y}{d t}=-\frac{1}{2} a \rho S^{*} H_{I_{1}^{\star}}\left[a \rho S^{*} H_{s^{*} s^{*}}+2 a \rho H_{s^{*}}\right] x^{2}+f_{6}(x, y)
\end{aligned}
$$

where, $\mathrm{f}_{5}(\mathrm{X}, \mathrm{Y})$ and $\mathrm{f}_{6}(\mathrm{X}, \mathrm{Y})$ are $\mathrm{C}^{\propto}$ functions at least of order three. In order to find the canonical normal form of the cusp, we take:

$$
\mathrm{X}=\mathrm{x} \text { and } \mathrm{Y}=\mathrm{y}-\frac{1}{2}\left[a \rho S^{*} \mathrm{H}_{\mathrm{s}^{*} \mathrm{~s}^{*}}+2 \mathrm{a} \rho \mathrm{H}_{\mathrm{s}^{*}}\right] \mathrm{x}^{2}+\mathrm{f}_{5}(\mathrm{x}, \mathrm{y})
$$

then the system (28) becomes Eq. 29:

$$
\begin{aligned}
& \frac{d X}{d t}=Y, \\
& \frac{d Y}{d t}=-\frac{1}{2} a \rho S^{*} H_{I_{1}^{\star}}\left[a \rho S^{*} H_{s^{*} s^{*}}+2 a \rho H_{s^{*}}\right] X^{2} \\
& -\left[a \rho S^{*} H_{s^{*} s^{*}}+2 a \rho H_{s^{*}}\right] X Y+f_{7}(X, Y)
\end{aligned}
$$

where, $\mathrm{f}_{7}(\mathrm{X}, \mathrm{Y})$ is $\mathrm{C}^{\propto}$ functions at least of order three. Now, since $\left(a \rho S^{*} H_{S^{*} S^{*}}+2 a \rho H_{S^{*}}\right)>0$, hence $\mathrm{P}_{2}=\left(\mathrm{S}^{*}\right.$, $\left.\mathrm{I}_{1}{ }_{1}\right)$ is a cusp of codimension 2. This completes the proof.

The above result indicates that Eq. 24 can satisfies the Bogdanov-Takens bifurcation with a small perturbation if the bifurcation parameters are chosen by suitable method. For convenience, we denote:

$$
\beta^{\circ}=\left(\alpha_{1}, \mathrm{a}_{1}, \mathrm{~d}_{1}, \gamma_{1}, \mathrm{k}_{11}\right) \text { and } \beta=\left(\alpha, \mathrm{a}, \mathrm{d}, \gamma, \mathrm{k}_{1}\right) \text {. }
$$

Then the system (2) in a small neighborhood of $\left(\mathrm{S}^{*}, \mathrm{I}_{1}\right)$ can be written as Eq. 30:

$$
\begin{aligned}
& \frac{d x}{d t}=y+W_{1}(x, y ; \beta), \\
& \frac{d y}{d t}=-\frac{1}{2} a \rho S^{*} H_{I_{1}^{*}}\left[a \rho S^{*} H_{s^{*} s^{*}}+2 a \rho H_{s^{*}}\right] x^{2} \\
& +\left[a \rho S^{*} H_{s^{*} s^{*}}+2 a \rho H_{s^{*}}\right] x y+W_{2}(x, y ; \beta)
\end{aligned}
$$

where, $\mathrm{W}_{1}$ and $\mathrm{W}_{2}$ are $\mathrm{C}^{\propto}$ functions, $\mathrm{W}_{1}\left(\mathrm{x}, \mathrm{y} ; \beta^{\circ}\right)=0$, $\mathrm{W}_{2}(\mathrm{x}, \mathrm{y} ; \beta)=\mathrm{f}_{7}(\mathrm{X}, \mathrm{Y}), \mathrm{x}$ and $\mathrm{y}$ belong to small neighborhood of $(0,0)$ and $\beta$ is a small neighborhood of $\beta^{\circ}$. Next we obtain versal unfolding depending on the original parameters in Eq. 4. By this method,we will compute the approximating bifurcations curves. As the bifurcation parameters, we can choose the parameters a, $\mathrm{d}$ and $\rho$. Assume that the following assumption holds:

$$
\left(\mathrm{A}_{2}\right) \quad \mathrm{H}_{\mathrm{I}_{1}^{\star} 1_{1}^{\star}}=0
$$

Assume that a, $d$ and $\rho$ satisfy $\left(\mathrm{A}_{1}\right)$ and $\left(\mathrm{A}_{2}\right)$. Let a $=a_{\circ}+\mu_{1}$ and $d=d_{\circ}+\mu_{2}$ and $\rho=\rho_{\circ}+\mu_{3}$. Substituting $X=$ S-S ${ }^{*}$ and $\mathrm{Y}=\mathrm{I}-\mathrm{I}_{1}{ }_{1}$ into (24) and using Taylor expansion, we obtain Eq. 31:

$$
\begin{aligned}
& \frac{d x}{d t}=a_{\circ}+a_{1} x+a_{2} y+a_{3} x^{2}+a_{4} x y+p_{1}(x, y), \\
& \frac{d y}{d t}=b_{0}+b_{1} x+b_{2} y+b_{3} x^{2}+b_{4} x y+p_{2}(x, y)
\end{aligned}
$$

Where:

$$
\begin{aligned}
& \mathrm{a}_{\circ}=\alpha d-a \rho S^{*} H^{*}-d S^{*}, \quad a_{1}=-\left(a \rho H^{*}+2 a \rho S^{*} H_{s^{*}}+d\right) \\
& a_{2}=-a \rho S^{*} H_{I_{1}^{\star}} a_{3}=\frac{1}{2}\left[-2 a \rho H_{s^{*}}-a \rho S^{*} H_{s^{*} s^{*}}\right] \\
& a_{4}=-\left[a \rho H_{I_{1}^{\star}}+a \rho S^{*} H_{s^{*} I_{1}^{\star}}\right], b_{0}=a \rho S^{*} H^{*}-\left(d+k_{1}-\gamma\right) I_{1}^{\star} \\
& b_{1}=a \rho S^{*} H_{s^{*}}+a \rho H^{*}, b_{2}=a \rho H_{\mathrm{I}_{1}^{\star}}-\left(d+k_{1}\right), \\
& b_{3}=\frac{1}{2}\left[a \rho S^{*} H_{s^{*} s^{*}}+2 a \rho H_{s^{*}}\right], b_{4}=a \rho H_{S^{*} I_{1}^{\star}}+a \rho S^{*} H_{1_{1}^{\star}}
\end{aligned}
$$

and $\mathrm{p}_{1}(\mathrm{x}, \mathrm{y})$ and $\mathrm{p}_{2}(\mathrm{x}, \mathrm{y})$, are $\mathrm{C}^{\propto}$ functions of $(\mathrm{x}, \mathrm{y})$ up to the third order. Using the change of variables:

$$
X=x \text { and } Y=a_{0}+a_{1} x+a_{2} y+a_{3} x^{2}+a_{4} x y+p_{1}(x, y)
$$

and rewrite $\mathrm{X}, \mathrm{Y}$ as $\mathrm{x}, \mathrm{y}$ respectively, system (31) becomes Eq. 32:

$$
\begin{aligned}
& \frac{d x}{d t}=y \\
& \frac{d y}{d t}=a_{5}+a_{6} x+a_{7} y+a_{8} x^{2}+a_{9} y^{2}+a_{10} x y+p_{3}(x, y, \mu)
\end{aligned}
$$

Where:

$$
\begin{aligned}
& a_{5}=a_{\circ} a_{1}+a_{2} b_{0}, \\
& a_{6}=a_{1}^{2}+a_{2} b_{1}+2 a_{0} a_{3}+a_{4} b_{0}, \\
& a_{7}=a_{1} a_{2}+a_{2} b_{2}+a_{\circ} a_{4}, \\
& a_{8}=a_{1} a_{3}+a_{2} b_{3}+2 a_{1} a_{3}+a_{4} b_{1}, \\
& a_{9}=a_{2} a_{4}, \quad a_{10}=a_{1} a_{4}+a_{2} b_{4}+2 a_{2} a_{3}+a_{4} b_{2}
\end{aligned}
$$

and $\mathrm{p}_{3}$ is a continous function. Let $\mathrm{X}=\mathrm{x}+\frac{\mathrm{a}_{7}}{\mathrm{a}_{10}}$ and rewriting $\mathrm{X}$ as $\mathrm{X}$, we obtain Eq. 33: 
Am. J. Applied Sci., 9 (7): 961-967, 2012

$\frac{\mathrm{dx}}{\mathrm{dt}}=\mathrm{y}$,

$\frac{d y}{d t}=b_{5}+b_{6} x+a_{8} x^{2}+a_{9} y^{2}+a_{10} x y+p_{4}(x, y, \mu)$

where $b_{5}=\left(a_{5}-\frac{a_{6} a_{7}}{a_{10}}+\frac{a_{8} a_{7}^{2}}{a_{10}^{2}}\right), b_{6}=\left(a_{6}-\frac{2 a_{7} a_{8}}{a_{10}}\right) \quad$ and

$\mathrm{p}_{4}(\mathrm{x}, \mathrm{y}, \mu)$ is a continous function in the variables $\mathrm{x}, \mathrm{y}$ and the parameter $\mu$. Now, introduce the new time $\tau$ by $\mathrm{dt}=\left(1-\mathrm{a}_{1} \mathrm{x}\right) \mathrm{d} \tau$ and rewrite $\tau$ as $\mathrm{t}$, we have Eq. 34 :

$\frac{d x}{d t}=\left(1-a_{1} x\right) y$,
$\frac{d y}{d t}=\left(1-a_{1} x\right)\left(b_{5}+b_{6} x+a_{8} x^{2}+a_{9} y^{2}+a_{10} x y+p_{4}(x, y, \mu)\right)$,

Set $\mathrm{X}=\mathrm{x}$ and $\mathrm{Y}=\left(1-\mathrm{a}_{1} \mathrm{x}\right) \mathrm{y}$ and rename $\mathrm{X}, \mathrm{Y}$ as $\mathrm{x}$, y respectively, we obtain Eq. 35:

$$
\begin{aligned}
& \frac{d x}{d t}=y, \\
& \frac{d y}{d t}=b_{5}+C_{1} x+C_{2} x^{2}+a_{10} x y+p_{5}(x, y, \mu),
\end{aligned}
$$

where, $C_{1}=b_{6}-2 a_{9} b_{5}, C_{2}=a_{8}-2 a_{9} b_{6}$ and $p_{5}(x, y, \mu)$ is a smooth function of in xy-plane and $\mu$ at least of third order. Setting the change of variables $X=\frac{a_{10}^{2}}{C_{2}} x, \quad Y=\frac{a_{10}^{3}}{C_{2}^{2}} y, \quad \tau=\frac{C_{2}}{a_{10}} t$. Also, we denote them again by $x, y$ and $t$, respectively, we get Eq. 36:

$$
\begin{aligned}
& \frac{d x}{d t}=y, \\
& \frac{d y}{d t}=\xi_{1}+\xi_{2} x+x^{2}+x y+p_{6}(x, y, \mu),
\end{aligned}
$$

where $\xi_{1}=\frac{b_{5} a_{10}^{4}}{C_{2}^{3}}, \xi_{2}=\frac{a_{10}^{2} C_{1}}{C_{2}^{2}}$ and $p_{6}(x, y, \mu)$ is a continous function in the variables $\mathrm{x}, \mathrm{y}$ and the parameter $\mu$. As in (Bogdanov, 1981a; 1981b), we will get the following bifurcation curves.

Theorem 3.2: Suppose $\left(A_{1}\right)$ and $\left(A_{2}\right)$ hold. Then system (24) satisfies the following bifurcation curves:

- The saddle-node bifurcation curve $\mathrm{SN}=\left\{\left(\xi_{1}, \xi_{2}\right): \xi_{1}=\frac{1}{4} \xi_{2}^{2}\right\}$

- The Hopf bifurcation curve $\mathrm{H}=\left\{\left(\xi_{1}, \xi_{2}\right): \xi_{1}=0, \xi_{2}\right.$ $<0\}$
- The homoclinic bifurcation curve

$$
\mathrm{HL}=\left\{\left(\xi_{1}, \xi_{2}\right): \xi_{1}=\frac{-6}{25} \xi_{2}^{2}+0\left(\|\xi\|^{2}\right)\right\}
$$

\section{CONCLUSION}

In this study, we considered a general HIV/AIDS system with treatment model. The incidence rates $\rho \mathrm{H}$ $\left(\mathrm{I}_{1}, \mathrm{I}_{2}\right)$ and $\rho \mathrm{H}\left(\mathrm{S}, \mathrm{I}_{1}\right)$ are of nonlinear form. The local asymptotic stability of the disease-free equilibrium points $\overline{\mathrm{P}}_{\circ}=(\alpha, 0,0)$ and $\mathrm{P}^{*}=\left(\mathrm{S}^{*}, \mathrm{I}^{*}{ }_{1}, \mathrm{I}_{2}{ }_{2}\right)$ for systems (1) and (2), respectively are established. The obtained results in here are consistent with those obtained by (Perelson and Nelson, 1999). We proved that The

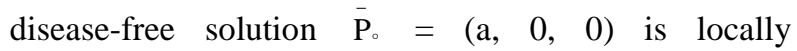
asymptotically stable in the interior of the feasible region and the disease always dies out. Also we showed that the non-trivial equilibrium point $\mathrm{P}^{*}=\left(\mathrm{S}^{*}, \mathrm{I}_{1}{ }_{1}, \mathrm{I}_{2}{ }_{2}\right)$ exists and is locally asymptotically stable in the considered region. In Theorem 3.1 we proved that if the two conditions $\left(A_{1}\right)$ and $\left(A_{2}\right)$ hold, then the equilibrium point $\mathrm{P}_{2}=\left(\mathrm{S}^{*}, \mathrm{I}_{1}{ }_{1}\right)$ of system (24) is a cusp of codimension 2, i,e., it is a Boggdanov-Takens singularity. Also we have proved that if the additional condition $\left(\mathrm{A}_{2}\right)$ holds, then the system (24) exhibits Boggdanov-Takens bifurcation, that is, there are three types of bifurcations, saddle-node bifurcation, Hopf bifurcation and homoclinic bifurcation. Our results that obtained througout this study are considered as improvement and partial generalization for those obtained by (Anderson, 1988; Busenberg and Driessche, 1990; Pedro and Tchuenche, 2010; Wang and Li, 2006; Xu, 2011; Yang and Xia, 2010).

\section{ACKNOWLEDGMENT}

This study is supported by Deanship of Scientific Research, Taibah University, Kingdom of Saudi Arabia No. (202-432).

The researcher is deeply indebted to the Professor Dr. M. M. A. El-Sheikh, the professor of pure mathematics at Menoufia University, Faculty of Science, Department of Mathematics for his valuable discussions and critical comments and ideas that help me to finalize this study.

\section{REFERENCES}

Anderson, R.M., 1988. The role of mathematical models in the study of HIV transmission and the epidemiology of AIDS. J. Acquir. Immune Defic. Syndr., 1: 241-256. PMID: 3216309 
Bachar, M. and A. Dorfmayr, 2004. HIV treatment models with time delay. Comptes Rendus Biol., 327: 983-994. DOI: 10.1016/j.crvi.2004.08.007

Bogdanov, R.I., 1981a. Versial deformations of a singular point on the plane in the case of zeroeigenvalues. Selecta Math. Sovit, 1: 4-4.

Bogdanov, R.I., 1981b. Bifurcation of the Limit cycle of a family of plane vector fields, Selecta Math. Sovit.

Busenberg, S. and P.V.D. Driessche, 1990. Analysis of a disease transmission model in a population with varying size. J. Math. Biol., 28: 257-270. DOI: 10.1007/BF00178776

Cai, L., X. Li, M. Ghosh and B. Guo, 2009. Stability analysis of an HIV/AIDS epidemic model with treatment. J. Comput. Applied Math., 229: 313323. DOI: 10.1016/j.cam.2008.10.067

Culshaw, R.V. and S. Ruan, 2000. A delay-differential equation model of HIV infection of CD4+ T-cells. Math. Biosci., 165: 27-39. DOI: 10.1016/S00255564(00)00006-7

Elaiw, A.M., 2010. Global properties of a class of HIV models. Nonlinear Anal.: Real World Appli., 11: 2253-2263. DOI: 10.1016/j.nonrwa.2009.07.001

El-Marouf, S.A.A. and S.M. Alihaby, 2011. Global analysis of an epidemic model with non-linear incidence rate. J. Math. Stat., 7: 319-325. DOI: 10.3844/jmssp.2011.319.325

Hethcote, H.W. and J.W.V. Ark, 1992. Modeling HIV Transmission and AIDS in the United States. 1st Edn., Springer-Verlag, Berlin, ISBN: 3540559043 , pp: 234.

Hethcote, H.W., M.A. Lewis, P.V.D. Driessche, 1989. An epidemiological model with a delay and a nonlinear incidence rate. J. Math. Biol., 27: 49-64. PMID: 2708918

Hsieh, Y.H. and C.H. Chen, 2004. Modelling the social dynamics of a sex industry: Its implications for spread of HIV/AIDS, Bull. Math. Biol., 66: 143-166. PMID: 14670534
LaSalle, J.P., 1976. The Stability of Dynamical Systems. 2nd Edn., SIAM, Philadelphia, PA., ISBN: 0898710227, pp: 76.

Mann, J.M. and D.J.M. Tarantola, 1998. HIV 1998: The global picture. Scientific Am., 279: 82-83.

McCluskey, C.C., 2003. A model of HIV/AIDS with staged progression and amelioration. Math. Biosci., 18: 1-16. DOI: 10.1016/S0025-5564(02)00149-9

Moghadas, S.M. and A.B. Gumel, 2003. A mathematical study of a model for childhood diseases with non-permanent immunity. J. Comput. Applied Math., 157: 347-363. DOI: 10.1016/S0377-0427(03)00416-3

Naresh, R., A. Tripathi and D. Sharma, 2011. A nonlinear AIDS epidemic model with screening and time delay. Applied Math. Comput., 217: 4416-4426. DOI: 10.1016/j.amc.2010.10.036

Pedro, S.A. and J.M. Tchuenche, 2010. HIV/AIDS dynamics: Impact of economic classes with transmission from poor clinical settings. J. Theoretical Biol., 267: 21 471-485. DOI: 10.1016/j.jtbi.2010.09.019

Perelson, A.S. and P.W. Nelson, 1999. Mathematical analysis of HIV-I: Dynamics in vivo. SIAM Rev., 41: 3-44.

Rao, M.R.M., 1981. Ordinary Differential Equations: Theory and Applications. 1st Edn., Edward Arnold London, ISBN: 0713134526, pp: 266.

Wang, L. and M.Y. Li, 2006. Mathematical analysis of the global dynamics of a model for HIV infection of CD4+ T-cells. Math. Biosci., 200: 44-57. DOI: 10.1016/j.mbs.2005.12.026

$\mathrm{Xu}, \mathrm{R} ., 2011$. Global stability of an HIV-1 infection model with saturation infection and intracellular delay. J. Math. Anal. Appli., 375: 75-81. DOI: 10.1016/j.jmaa.2010.08.055

Yang, Y. and Y. Xia, 2010. Threshold dynamics for an HIV model in periodic environments. J. Math. Anal. Appli., 361: 59-68. DOI: 10.1016/j.jmaa.2009.09.012 